\title{
A techniques for recognition of a human faces on eye with python
}

\author{
Vu Ngoc Phan ${ }^{1}$, Nguyen Duc Toan ${ }^{1}$ \\ Hanoi University of Natural Resources and Environment, Vietnam ${ }^{1}$
}

\begin{abstract}
Human face recognition is a field of study in Computer Vision, and is also considered a research area of Biometrics (similar to fingerprint recognition, or iris recognition). In terms of general principles, facial recognition has a great resemblance to fingerprint recognition and iris recognition, but the difference lies in the specific extraction step of each field. While fingerprint and iris recognition has reached maturity, which is widely applicable in practice, facial recognition remains challenging and remains an interesting area of research with many. people. Compared to fingerprint and iris recognition, facial recognition has a richer data source (you can see human faces in any photo or video clip related to people online) and requires less more controlled interaction (to perform fingerprint or iris recognition, human input requires cooperation in a controlled environment).

Currently, the face recognition methods are divided into many directions according to different criteria: still image based FR (2D) recognition is the most popular, but the future will probably be. 3D (because the layout of many 2D cameras will give 3D data and give better and more reliable results), it can also be divided into two directions: doing with image data and doing with video data.
\end{abstract}

Keywords: Human faces, iris recognition, facial recognition, fingerprint recognition

\section{INTRODUCTION}

The eye is the most significant features in a human face. Generally eye detection, face detection and iris detection are used in person identification and security system. For eye detection firstly detect face part of human body. face detection and recognitions are also play important role. And they has many application like interface, surveillance, security. in this paper, face, eye and iris part detect in lively image.

It is known that eye regions are usually darker than face part. there fore in this we have to set threshold value. Generally human eye is $10 \%$ of face part. Recently many eye detection technique have been reported, Employed size and intensity information to find eye-analog segments from a gray scale image, and exploited the special geometrical relationship to filter out the possible eye-analogy pairs[1]. Iris is a circular part of the eye. it is most sensitive organ of human body. there are many application used based on iris. The function of the iris is to control the amount of light entering through the pupil. The characteristic of iris which can be used in personal identification and security. The average diameter of the iris is $12 \mathrm{~mm}$, and the pupil size can vary from $10 \%$ to $80 \%$ of the iris diameter [7]. The

Iris recognition techniques potentially prevent unauthorised access to ATM, security, personal Identification etc. the Accuracy of iris is much hither compares to other technique like fingerprint, voiceprint etc. there are several methods like integro differential operator, Hough transform, gradient based edge detection are used to localize the portion of iris and pupil from live eye image. These methods are based on radius, gradient, probability and moments. wildes proposed a circular Hough transform to detect eyelid, upper and lower threshold. this requires an appropriate threshold value[8].Daugman proposed an infero differential operator to find pupil, iris and eyelid[9].

In this paper, we proposed a method for automatic eye and face detection of human and also detect circular pattern i.e Iris of cropped eye image. Blob analysis is used for eye and face part detection in live image.

\section{FACE AND EYE DETECTION}

To capture the rich details of iris patterns, an imaging system should resolve a minimum of 70 pixels in iris radius. In the field trials to date, a resolved iris radius of 100 to 140 pixels has been more typical. Monochrome CCD cameras ( $480 \mathrm{x}$ 640) have been used because NIR illumination in the 700nm - 900nm band was required for imaging to be invisible to humans. Some imaging platforms deployed a wideangle camera for coarse localization of eyes in faces, to steer the optics of a narrow-angle pan/tilt camera that acquired higher resolution images of eyes. There exist many alternative methods for finding and tracking facial features such as the eyes, and this wellresearched topic will not be discussed further here. In these trials, most imaging was done without active pan/tilt camera optics, but instead exploited visual feedback via a mirror or video image to enable cooperating Subjects to position their own eyes within the field of view of a single narrow-angle camera.

Focus assessment was performed in real-time (faster than video frame rate) by measuring the total high-frequency power in the 2D Fourier spectrum of each frame, and seeking to maximize this quantity either by moving an active lens or by providing audio feedback to Subjects to adjust their range appropriately. Images passing a minimum focus criterion were 


\section{International Journal of Advanced Research in Computer and Communication Engineering}

Vol. 10, Issue 8, August 2021

DOI 10.17148/IJARCCE.2021.10822

then analyzed to find the iris, with precise localization of its boundaries using a coarse-to-fine strategy terminating in single-pixel precision estimates of the center coordinates and radius of both the iris and the pupil. Although the results of the iris search greatly constrain the pupil search, concentricity of these boundaries cannot be assumed. Very often the pupil center is nasal, and inferior, to the iris center. Its radius can range from 0.1 to 0.8 of the iris radius. Thus, all three parameters defining the pupillary circle must be estimated separately from those of the iris.

A very effective integrodifferential operator for determining these parameters is:

$$
\operatorname{MAX}\left(r, x_{0}, y_{0}\right)\left|G_{\sigma}(r) * \frac{\partial}{\partial_{r}} \oint_{r, x_{0}, y_{0}} \frac{I(x, y)}{2 \pi r} d s\right|(1)
$$

Where $\mathrm{G} \sigma(\mathrm{r})$ is a smoothing function and $\mathrm{I}(\mathrm{x}, \mathrm{y})$ is the image of the eye. All early research in iris segmentation assumed that the iris had a circular boundary. However, often the pupillary and limbic boundaries are not perfectly circular. Recently, Daugman has studied alternative segmentation techniques to better model the iris boundaries [2]. Even when the inner and outer boundaries of the iris are found, some of the iris still may be occluded by eyelids or eyelashes.

The distance from the camera affects the size of the iris in the image. Also, changes in illumination can cause the iris to dilate or contract. This problem was addressed by mapping the extracted iris region into a normalized coordinate system. To accomplish this normalization, every location on the iris image was defined by two coordinates, (i) an angle between 0 and 360 degrees, and (ii) a radial coordinate that ranges between 0 and 1 regardless of the overall size of the image. This normalization assumes that the iris stretches linearly when the pupil dilates and contracts. A paper by Wyatt [3] explains that this assumption is a good approximation, but it does not perfectly match the actual deformation of an iris. The normalized iris image can be displayed as a rectangular image, with the radial coordinate on the vertical axis, and the angular coordinate on the horizontal axis. In such a representation, the pupillary boundary is on the bottom of the image, and the limbic boundary is on the top. The left side of the normalized image marks 0 degrees on the iris image, and the right side marks 360 degrees. The division between 0 and 360 degrees is somewhat arbitrary, because a simple tilt of the head can affect the angular coordinate. Daugman accounts for this rotation later, in the matching technique. Directly comparing the pixel intensity of two different iris images could be prone to error because of differences in lighting between two different images.

Daugman uses convolution with 2-dimensional Gabor filters to extract the texture from the normalized iris image. In his system, the filters are "multiplied by the raw image pixel data and integrated over their domain of support to generate coefficients which describe, extract, and encode image texture information." [4] After the texture in the image is analyzed and represented, it is matched against the stored representation of other irises. If iris recognition were to be implemented on a large scale, the comparison between two images would have to be very fast. Thus, Daugman chose to quantize each filter's phase response into a pair of bits in the texture representation. Each complex coefficient was transformed into a two-bit code: the first bit was equal to 1 if the real part of the coefficient was positive, and the second bit was equal to 1 if the imaginary part of the coefficient was positive. Thus after analyzing the texture, of the image using the Gabor filters, the information from the iris image was summarized in a 256 byte (2048 bit) binary code. The resulting binary "iris codes" can be compared efficiently using bitwise operations.

Daugman uses a metric called the normalized Hamming distance, which measures the fraction of bits for which two iris codes disagree. A low normalized Hamming distance implies strong similarity of the iris codes. If parts of the irises are occluded, the normalized Hamming distance is the fraction of bits that disagree in the areas that are not occluded on either image. To account for rotation, comparison between a pair of images involves computing the normalized Hamming distance for several different orientations that correspond to circular permutations of the code in the angular coordinate. The minimum computed normalized Hamming distance is assumed to correspond to the correct alignment of the two images. The modules of an iris biometrics system generally following Daugman's approach are depicted in the goal of segmentation is to isolate the region that represents the iris.

The goal of texture analysis is to derive a representation of the iris texture that can be used to match two irises. The goal of matching is to evaluate the similarity of two iris representations. The distinctive essence of Daugman's approach lies in conceiving the representation of the iris texture to be a binary code obtained by quantizing the phase response of a texture filter. This representation has several inherent advantages. Among these are the speed of matching through the normalized Hamming distance, easy handling of rotation of the iris, and an interpretation of the matching as the result of a statistical tes of independence [5].

\section{IRIS DETECTION}

\subsection{Proposed chart}

Human Iris is very important organ in human body. in this paper iris is detected in all direction ( left, right, up, down). in above section we shown detection of eye and face part in real time, once we detect face and eye part after that we will detect iris in different positions. in this paper circular. Hough transform is used to detect iris part in cropped eye images. The Hough transformation is a procedure generally used to compute the parameters of the geometri objects such as lines 


\section{International Journal of Advanced Research in Computer and Communication Engineering}

Vol. 10, Issue 8, August 2021

DOI 10.17148/IJARCCE.2021.10822

and circles in an image. For detecting the center coordinates and radius of the iris and pupil regions, the circular Hough transform can be used. This technique generally uses a voting procedure to find shapes of the objects within the classes available. The Hough segmentation algorithm firstly creates an edge map by computing the gradients or first derivatives of intensity values in an eye. The center coordinates and the radius can be found using the following equation:

$x_{c}^{2}+y_{c}^{2}-r^{2}=0$

In the Hough space, the maximum point corresponds to the center coordinates (xc,yc) and the radius ' $r$ ' of the circle is given by the edge points. When performing the edge detection, we have considered derivatives/gradients in the vertical direction to detect the iris-sclera boundary to decrease the effect of the eyelids which are horizontally aligned (6). The vertical gradients are taken for locating the iris boundary and to reduce the influence of the eyelids. When performing circular Hough transform, not all of the edge pixels that define the circle are required for successful localization. Not only does this make circle localization more accurate, but it also makes it more efficient, since there are fewer edge points to cast votes in the Hough space (6).

Using equation 2 , the model transforms every pixel in the circular iris into an equivalent position on the polar axes $(r, \theta)$ where $r$ is the radial distance and $\theta$ is the rotated angle at the corresponding radius. The radial resolution describes the number of radial lines generated around the iris region while the angular resolution is the number of data points in the radial direction:

$$
\mathrm{I}[\mathrm{x}(\mathrm{r}, \theta), \mathrm{y}(\mathrm{r}, \theta)] \rightarrow I(r, \theta)
$$

The iris region is converted to a two-dimensional array. Horizontal dimension represents the angular resolution, and the vertical dimension represents radial resolution, where $I(x, y)$ corresponds to the iris region, $(x, y)$ and $(r, \theta)$ are the Cartesian and normalized polar coordinates, respectively. Ranges of $\theta$ from 0 to $2 \pi$ and $r$ from $R_{p}$ to $R_{l} . x(r, \theta)$ and $\mathrm{y}(\mathrm{r}, \theta)$ are defined as linear combinations of pupil boundary points.

\subsection{Recommended algorithm}

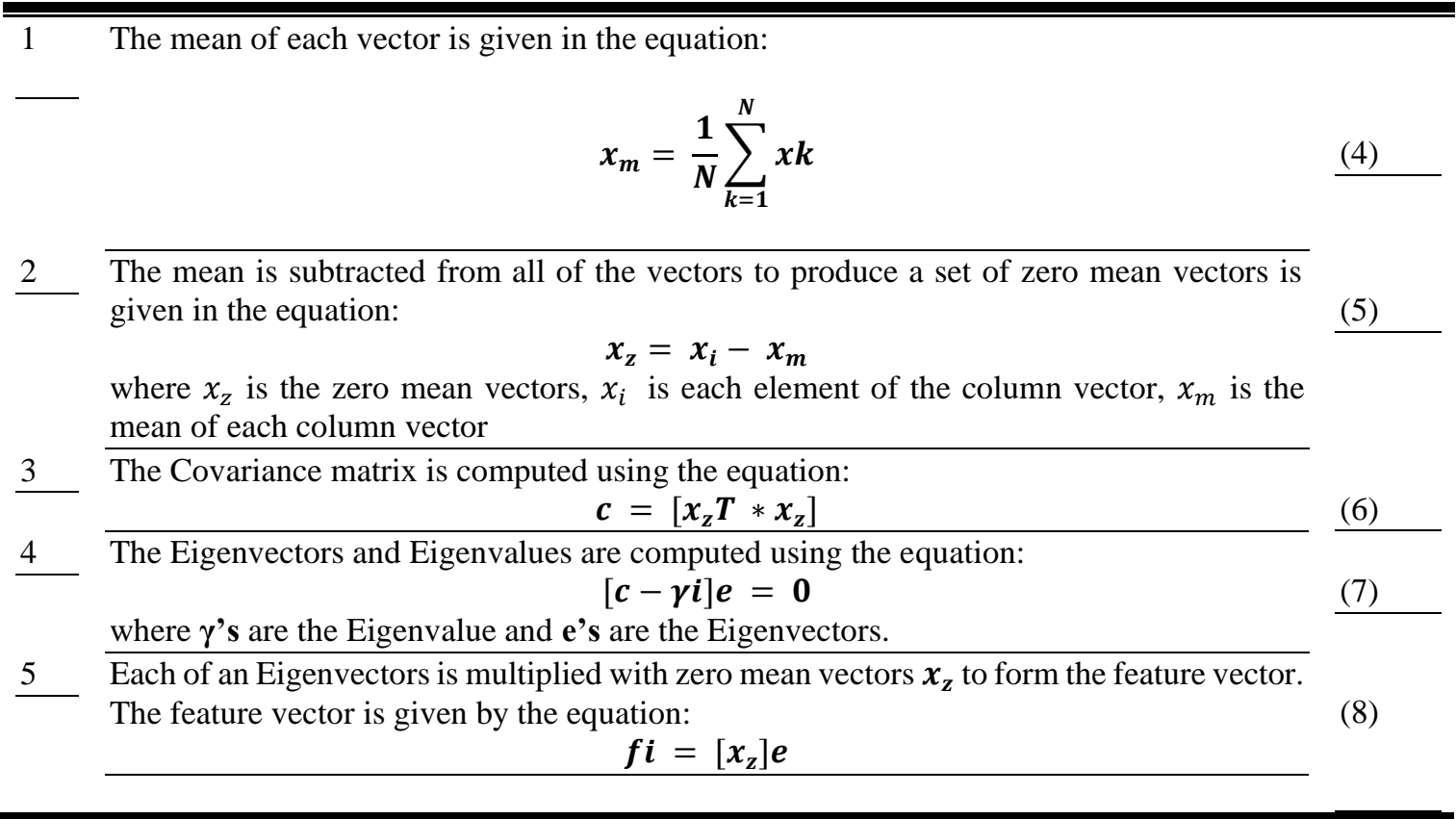

\section{EXPERIMENTAL RESULTS}

\subsection{Face detection result}

For our Experimental Result we have use intel core i5 CPU and Windows 10 operating system. we have uses 12 MP of Web Camera for images. automatic eye and face are detected automatic in real time images. 


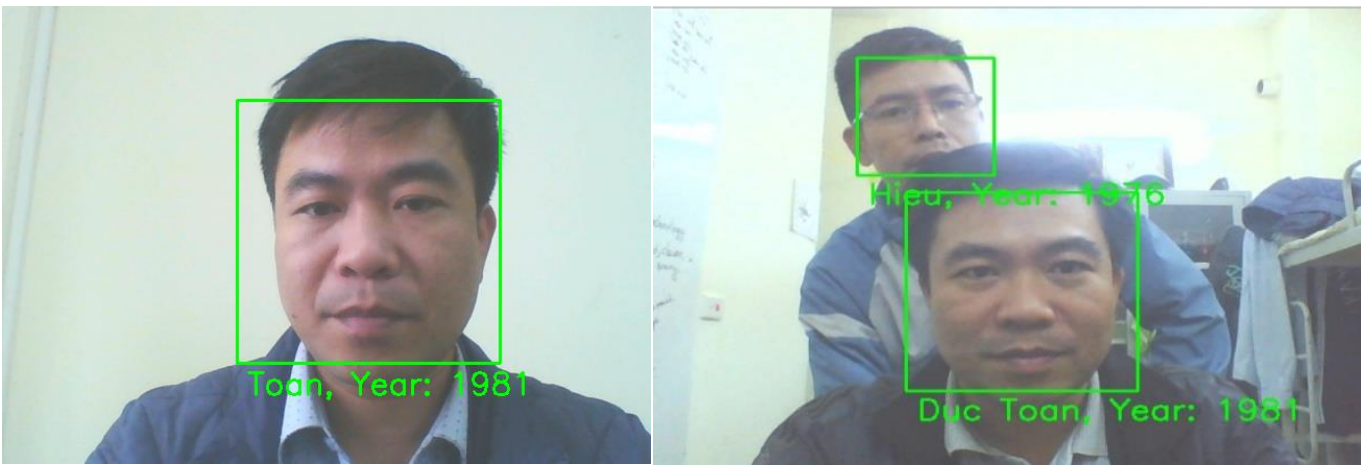

\subsection{Iris detection result}

Above section shows eye and face part detection. In this part shows iris detection in different positions. The experimental result shown below.

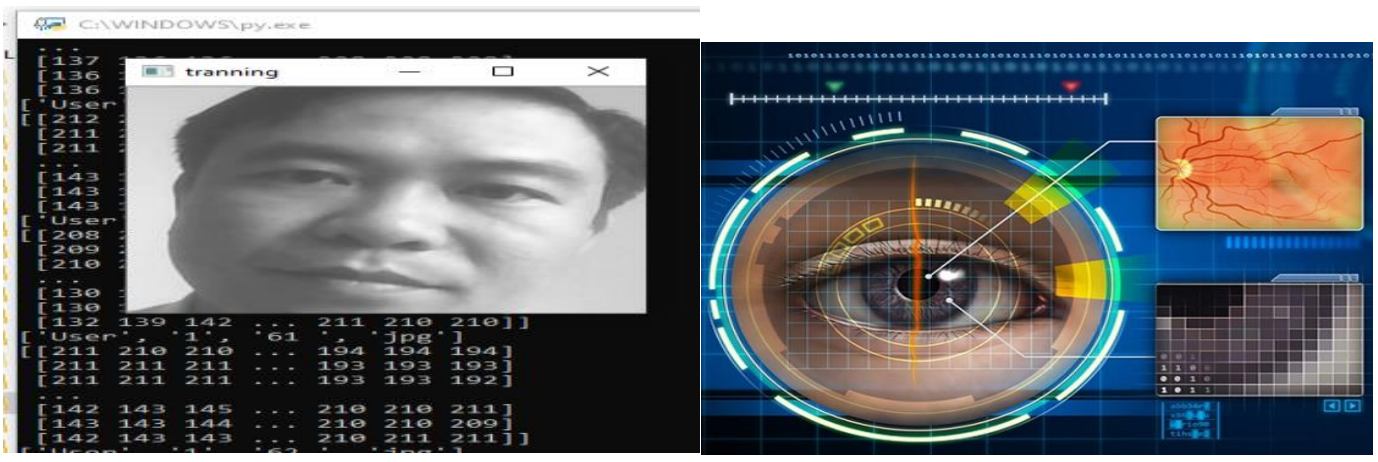

\section{CONCLUSION}

We have concluded that it can be automatic detect face and eye regions and also iris detection. We have used blob analysis and Hough transform for eye and iris detection resp. Blob analysis is based on skin detection. Blob analysis is very fast way to recognition faces and eye region. And it processing time is fast than other recognitions technique. we proposed an iris detection method using the circular Hough transform that adapts to various eye positions. Firstly detected the eyes in different position and cropped automatically. Then the positions of irises were detected by circular Hough transform. So I have concluded that Hough transform is very easily implement and also conceptually simple.

\section{REFERENCES}

[1]. Springer S. M. Metev and V. P. Veiko, Laser Assisted Microtechnology, 2nd ed., R. M. Osgood, Jr., Ed. Berlin, Germany: -Verlag, 1998.

[2]. John Daugman. New methods in iris recognition. IEEE Transactions on Systems, Man and Cybernetics - B, 37(5):1167-1175, Oct 2007.

[3]. Harry Wyatt. A minimum wear-and-tear meshwork for the iris. Vision Research, 40:2167-2176, 2000

[4]. John Daugman. Biometric personal identification system based on iris analysis. U.S. Patent No. 5,291,560, March 1994

[5]. John Daugman. High confidence visual recognition of persons by a test of statistical independence. IEEE Transactions on Pattern Analysis and Machine Intelligence, 15(11):1148-1161, November 1993.

[6]. Verma, P., Dubey, M., Basu, S., and Verma, P. (2012a). Hough transform method for iris recognition- a biometric approach. International Journal of Engineering and Innovative Technology (IJEIT), 1(6):43-48

[7]. R. anandmarugan, "Methodology for iris segmentation and recognition using multi- resolution transform", IEEE- IcoAc 2011

[8]. R. Wildes, "Iris Recognition: An Emerging Biometrics Technology", Proceeding of the IEEE, vol. 85, no.9 1997

[9]. J. Daughman, "New IEEE Method In Iris on, Recognition” Transsaction System, Man and Cybernectics-part , vol. 3 no. 75 2007 


\section{BIOGRAPHY}

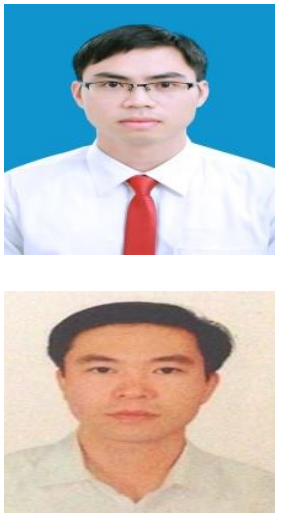

$\mathrm{Vu}$ Ngoc Phan is a Lecturer at Hanoi University of Natural Resources and Environment (Hanoi, Vietnam).

Graduated from University and Master degree at Hanoi University of Mining and Geology.Currently he is a PhD student, majoring in remote sensing and GIS His research interests include cryptography, communications, and network security.

Nguyen Duc Toan is a Lecturer at Hanoi University of Natural Resources and Environment (Hanoi, Vietnam).

Graduated from Hanoi Pedagogical University 2.

$\mathrm{PhD}$ in 2019 at University of Information and Communication Technology - Thai Nguyen University.

His research interests include cryptography, communications, and network security. 